Research Paper

\title{
Promotion of Transplanted Bone Marrow-derived Cell Migration into the Periodontal Tissues due to Ortho- dontic Mechanical Stress
}

\author{
Mihoko Tomida ${ }^{\circledR}$, Hidetsugu Tsujigiwa², Keisuke Nakano ${ }^{3}$, Rina Muraoka4, Takami Nakamura ${ }^{1}$, Nori- \\ masa Okafuji ${ }^{3}$, Hitoshi Nagatsuka², Toshiyuki Kawakami ${ }^{3}$ \\ 1. Department of Oral Physiology, Matsumoto Dental University School of Dentistry, Shiojiri, Japan \\ 2. Department of Oral Pathology and Medicine, Okayama University Graduate School of Medicine, Dentistry and Pharmaceutical Sciences, \\ Okayama, Japan \\ 3. Department of Hard Tissue Research, Matsumoto Dental University Graduate School of Oral Medicine, Shiojiri, Japan \\ 4. Department of Orthodontics, Matsumoto Dental University School of Dentistry, Shiojiri, Japan
}

$\square$ Corresponding author: Mihoko Tomida, PhD, Associate Professor, Department of Oral Physiology, Matsumoto Dental University School of Dentistry, 1780 Hirooka-Gobara, Shiojiri, 399-0781 Japan. Phone: +81-(0)263-51-2053, Fax: +81-(0)263-51-2053, E-mail: mtomi@po.mdu.ac.jp

( ) Ivyspring International Publisher. This is an open-access article distributed under the terms of the Creative Commons License (http://creativecommons.org/ licenses/by-nc-nd/3.0/). Reproduction is permitted for personal, noncommercial use, provided that the article is in whole, unmodified, and properly cited.

Received: 2013.05.06; Accepted: 2013.07.22; Published: 2013.08.09

\begin{abstract}
Background: Bone marrow-derived cells (BMCs) have abilities of cell migration and differentiation into tissues/organs in the body and related with the differentiation of teeth or periodontal tissue including fibroblasts. Then, we examined the effect of orthodontic mechanical stress to the transplanted BMC migration into periodontal tissues using BMC transplantation model.

Material and Method: BMC from green fluorescence protein (GFP) transgenic mice were transplanted into 8-week-old female C57BL/6 immunocompromised recipient mice, which had undergone $10 \mathrm{~Gy}$ of lethal whole-body-irradiation. Five mice as experimental group were received orthodontic mechanical stress using separator between first molar (MI) and second molar (M2) I time per week for 5 weeks and 5 mice as control group were not received mechanical stress. The maxilla with $\mathrm{MI}$ and $\mathrm{M} 2$ was removed and was immunohistochemically analyzed using a Dako Envision + Kit-K4006 and a primary anti-GFP-polyclonal rabbit antibody. Immunohistochemically stained was defined as positive area and the pixel number of positive area in the periodontal tissue was compared with the previously calculated total pixel number of the periodontal tissue.

Results: The immunohistochemistry revealed that GFP positive cells were detected in the periodontal tissues, both in the experimental and control specimens. The ratio of pixel number in the examination group showed $5.77 \pm 3.24 \%$ (mean \pm SD); and that in the control group, $0.7 \mathrm{I} \pm 0.45 \%$ (mean $\pm \mathrm{SD}$ ). The examination group was significantly greater than that of control group (Mann-Whitney $U$ test: $\mathrm{p}<0.00 \mathrm{I}$ ).

Conclusion: These results suggest that orthodontic mechanical stress accelerates transplanted BMC migration into periodontal tissues.
\end{abstract}

Key words: bone marrow-derived cell (BMC), periodontal tissue, green fluorescent protein (GFP), mechanical stress, fibroblast.

\section{Introduction}

It has been known that some kinds of stem cells have the remarkable properties of developing into a variety of cell types in the human body. Bone mar- row-derived cell (BMC), one of stem cells, has also multiple differentiation properties. Recently many researchers show that BMC might relate with various 
organs namely, retinal vessels, myoblasts, hepatocytes in the liver, Purkinje neurons, cardiac muscle in the heart, and airway epithelial cells [1,2]. This fact is very important because such cells could be used to regenerate organs for treatment of various diseases [3]. Nowadays, the local delivery of BMCs has been tried as a widely investigated tool for the treatment of ischemic disease including peripheral limb ischemia and myocardial infarction $[4,5]$.

In our previous study, we investigated the ability of BMC to distribute and differentiate into bone and tooth structures by transplantating bone marrow cells from green fluorescence protein (GFP) transgenic mice. GFP-positive cells were diffusely observed within the dental pulp of mouse incisor and in the periodontal ligaments, Langerhans cells in the oral epithelium, stromal fibroblasts, blood vessels and osteoclasts in the tooth region [6]. It was reported that tissue recombination of embryonic odontogenic epithelium with non-dental derived mesenchyme cells (neural stem cells, mouse embryonic stem cells and mouse BMCs) stimulates odontogenic response in stem cells, which could express odontogenic genes [7]. Applying traditional tissue engineering method, tooth-like structures can be produced from biodegradable polymer scaffolds seeded by dissociated tooth germs and direct cell pellet from dental pulp stem cells or BMC implantation [8].

On the other side, we established the proteins, BMP Runx2 and Msx2, expressed during orthodontic tooth movement inducing by mechanical stress. The BMP which has a role for bone forming state, Runx2 which induces differentiation of osteoblasts and Msx2 functioned as a promoting factor for Runx2 activity, maybe, strongly expressed at the tension side in the cells exposed to mechanical stress [9-11]. Regarding with tissue reaction occurring in orthodontic treatment, osteoblasts at surface of the tension side and osteoclasts at pressure side of relevant periodontal space of the alveolar bone appear, and play roles in resorption and addition of bone [12]. It has become clear that maintaining homeostasis can lead to periodontal tissue remodeling and expression of active molecules in response to various mechanical stress and inflammation [13]. However it is unclear that these proteins relate with BMC and the mechanical stress affects the migration of BMC. We investigated the migration of $\mathrm{BMC}$ in the periodontal tissue and the effect of orthodontic mechanical stress using bone marrow transplantation model.

\section{Materials and Methods}

\section{Animals and animal care}

Female GFP transgenic mice (C57BL/6-Tg
(CAG-EGFP)) [14] and female C57BL/6 mice were purchased from Okayama University Animal Center. All animals used in the present study were housed, supervised, and handled according to the Okayama University Graduate School of Medicine and Dentistry Guidelines for the Care and Use of Laboratory Animals. This research was approved by the Animal Experiment Control Committee of the Okayama University Graduate School of Medicine, Dentistry and Pharmaceutical sciences, under no. OKU-2010157.

\section{Bone marrow transplantation}

Bone marrow transplantation was carried out according to a standard protocol described previously [15]. Briefly, immediately after eight-week-old female C57BL/ 6 recipient mice had undergone 10 Gy of lethal whole-body-irradiation split into two doses separated by $5.0 \mathrm{~h}$ to minimize gastrointestinal toxicity, they were transplanted with bone marrow cells harvested from the femurs of GFP donors. Donor bone marrow cells were resuspended in Hank's balanced salt solution and $5 \times 106$ bone marrow cells were injected into the tail vein of the recipients. The recipient mice were maintained in a specific pathogen-free environment and received normal chow and hyper-chlorinated drinking water for the first 3 weeks after bone marrow transplantation. To determine the engraftment of the donor bone marrow, the bone marrow was examined 4 weeks after transplantation by immunohistochemical staining for GFP [16].

\section{Orthodontic mechanical stress}

The Waldo method of inducing mechanical stress-load in mouse periodontal tissues was applied to 5 mice [17]. Adjustable constant flow rate gas anesthesia system (Farmer Biometrical LTD., Laboratory, Osaka Japan) for small laboratory animals with isoflurane (Isoflu: Dainippon Sumitomo Pharma Co., Osaka, Japan) and gas-air mixture (4.0\% concentration of pre-anesthesia) in order to achieve a lasting and stable anesthesia was used. Under general anesthesia, the mouse was locked in a sitting position where the upper body was held on the experiment bench top. Isoflurane inhalation anesthesia was set to the nose for maintenance of general anesthesia $(1.0 \%$ concentration) during the experiment. To keep the mouth open, the maxilla was fixed with a thread tied on the upper incisor above the bench and rubber was used to fix the mandible. While the mouth was open, the separator was inserted to the maxillary molar to induce persistent mechanical stress by Waldo method. The separator was used with $2 \times 2 \mathrm{~mm}$ heavy force rubber dam sheet (Ivory, Premium rubber Dam Pure Latex: Heraeus Kulzer GmbH \& Co. KG, Hanau, 
Germany). Separator was inserted between first molar (M1) and second molar (M2) of right maxillary molars to ensure the mechanical stress due to pressure over a period of time $[18,19]$. The animals were divided into 2 groups: experimental $(n=5)$ and control $(n=5)$ groups. The experimental mice were received orthodontic mechanical stress 1 time per week for 5 weeks.

\section{Immunohistochemistry}

These experimental and control mice were sacrificed. The maxillary molar with the periodontal tissues were removed promptly and fixed in $4 \%$ paraformaldehyde with $0.05 \mathrm{M}$ phosphate buffer for 24 hours and decalcified in $10 \%$ EDTA solution for 3 weeks. After embedding in paraffin, serial sections of $5 \mu \mathrm{m}$ were deparaphinized and incubated for $30 \mathrm{~min}$ in $0.3 \% \mathrm{H}_{2} \mathrm{O}_{2}$ in methanol to block endogenous peroxidase. The sections were then incubated in $0.1 \%$ trypsin (Difco Laboratories, Detroit, Michigan) for 5 min at $37{ }^{\circ} \mathrm{C}$. After blocking of non-specific binding sites by incubation in $10 \%$ normal swine serum for 15 min, the sections were incubated overnight at $4{ }^{\circ} \mathrm{C}$ in anti-GFP polyclonal rabbit antibody (\#598; 1/500; MBL, Nagoya, Japan) at a dilution of 1:100. The sections were further incubated with anti-rabbit immunoglobulin antibody, Dako Emvision+Kit-K4006 (Dako, Glostrup, Denmark), for $30 \mathrm{~min}$. After rinsing in TBS, the sections were incubated at room temperature for $30 \mathrm{~min}$, followed by staining with $\mathrm{DAB}$ for 15 min.

\section{Analysis}

For semiquantitative evaluation of immunohistochemical staining, the following procedures were performed. First immunohistochemical images with same magnification from the related periodontal tissues of the distal buccal root of the maxillary first molar were prepared and pixel density was counted for each image. Then typical immunohistochemically positive staining part was defined as positive area. The pixel number of positive area in the periodontal tissue was compared with the previously calculated total pixel number of the periodontal tissue and the ratio was obtained. The statistical analysis was used Mann-Whitney U test.

\section{Results}

We examined the transplanted BMC migration into periodontal tissues. The immunohistochemistry revealed that GFP-positive cells were detected in the periodontal tissues, both in the experimental and control specimens. In the experimental group, there were numerous GFP-positive cells in the mechanically-stressed periodontal tissues (Fig.1A), especially tension side (Fig.1B). But there were few GFP-positive cells in the control specimens (Fig.1C, D). As a result of the experimental group specimens and control group, the stained areas in the periodontal tissues were analyzed. The ratio of pixel number in the experimental group showed $5.77 \pm 3.24 \%$ (mean $\pm \mathrm{SD}$ ); and that in the control group, $0.71 \pm 0.45 \%$ (mean \pm $\mathrm{SD}$ ). The experimental group was significantly greater than that of control group (Mann-Whitney $U$ test: $\mathrm{p}<0.001$ ) (Fig.2). Thus, these data indicated that orthodontic mechanical stress acts as a possible promoting factor of transplanted bone marrow-derived cell migration into periodontal tissues, and of differentiation to fibroblasts.

\section{Discussion}

Several varieties of stem cells, which comprise of two major classes: embryonic/fetal stem cells and adult stem cells, have been isolated and identified. Moreover, adult stem cells can be classified into several broad types such as hematopoietic stem cells, mesenchymal stem cells, liver stem cells and so on. These stem cells are capable of migrating to organs such as liver and brain after transplantation into immunocompromised mice [20,21]. All stem cells are equally important because of their versatility and pluripotentiality, which induces the stem cell therapy for the treatment of a variety of diseases in the human. Stem cell therapy has already reached the bedside in some hospitals the transplantation of donor bone marrow stem cells. It would be tremendously useful if embryonic, fetal, adult or umbilical cord stem cells could be coaxed to produce islets cells for the treatment of diabetes or neurons for neurodegenerative diseases, cardiomyocytes for heart disease, and so on $[22,23]$. It is hoped that it will play a major role in the treatment of a number of incurable diseases via transplantation therapy.

BMCs includes hematopoietic stem cells and mesenchymal stem cells. Hematopoietic stem cells is actively investigated as a means to stimulate the growth of new vasculature or enlargement of pre-existing vessels $[24,25]$ and have all been used to stimulate the restoration of blood flow in ischemic tissues via stimulation of new capillary growth or collateral vessel enlargement [26,27]. Mesenchymal stem cells (MSCs) are found postnatully in the nonhematopoietic bone marrow stroma. Marrow stromal tissue is made up of a heterogenous population of cells, which include reticular cells, adipocytes, osteogenic cells, smooth muscle cells, endothelial cells and macrophages [28]. In a steady state or in response to injury, turnover of stromal tissue and repair occurs through the participation of a population of stem cells found in the stromal tissue. Apart from bone marrow stroma, MSCs can also be derived from periosteum, 
fat cartilage, bone, muscle, tendon, ligament and fat [29]. There is some recent evidence that there is a rare cell within MSC cultures that is pluripotent and can give rise not only to mesodermal but to endodermal tissues [30]. MSCs are capable of differentiating into osteoblasts, chondrocytes or retinal cells, which means a transdifferentiation into cells of at least two different germ layers [20,31]. In recent times, dental cell therapies have been discussed by combining non-dental mesenchymal stem cells and dental stem cells $[7,8]$. A recent study demonstrated the positive effect of enamel matrix proteins on porcine bone marrow stem cells differentiation into cementoblasts [32].

Then many researchers focused on bone resorption and addition in the periodontal ligament (PDL) after mechanical stimulus used in orthodontic dental treatment [18]. Bone formation and resorption by showing existence of osteoclasts and osteoblasts, respectively, have been clarified. The power added to tooth, mechanical stress, induces tissue change in alveolar bone and PDL in the pressure and tension sides. In our experiment, the Msx2, Runx2, bone morphogenetic protein families (BMPs) and heat shock protein 27 (HSP27) exposed in the mouse PDL by mechanical stress. Run $x 2$ promoted differentiation of osteoblasts and Msx2 worked as an activator of Runx2 function [5]. BMPs relate with differentiation of osteoblasts and HSP27 acts as molecular chaperones for osteoblast activation and maintenance of homeostasis.

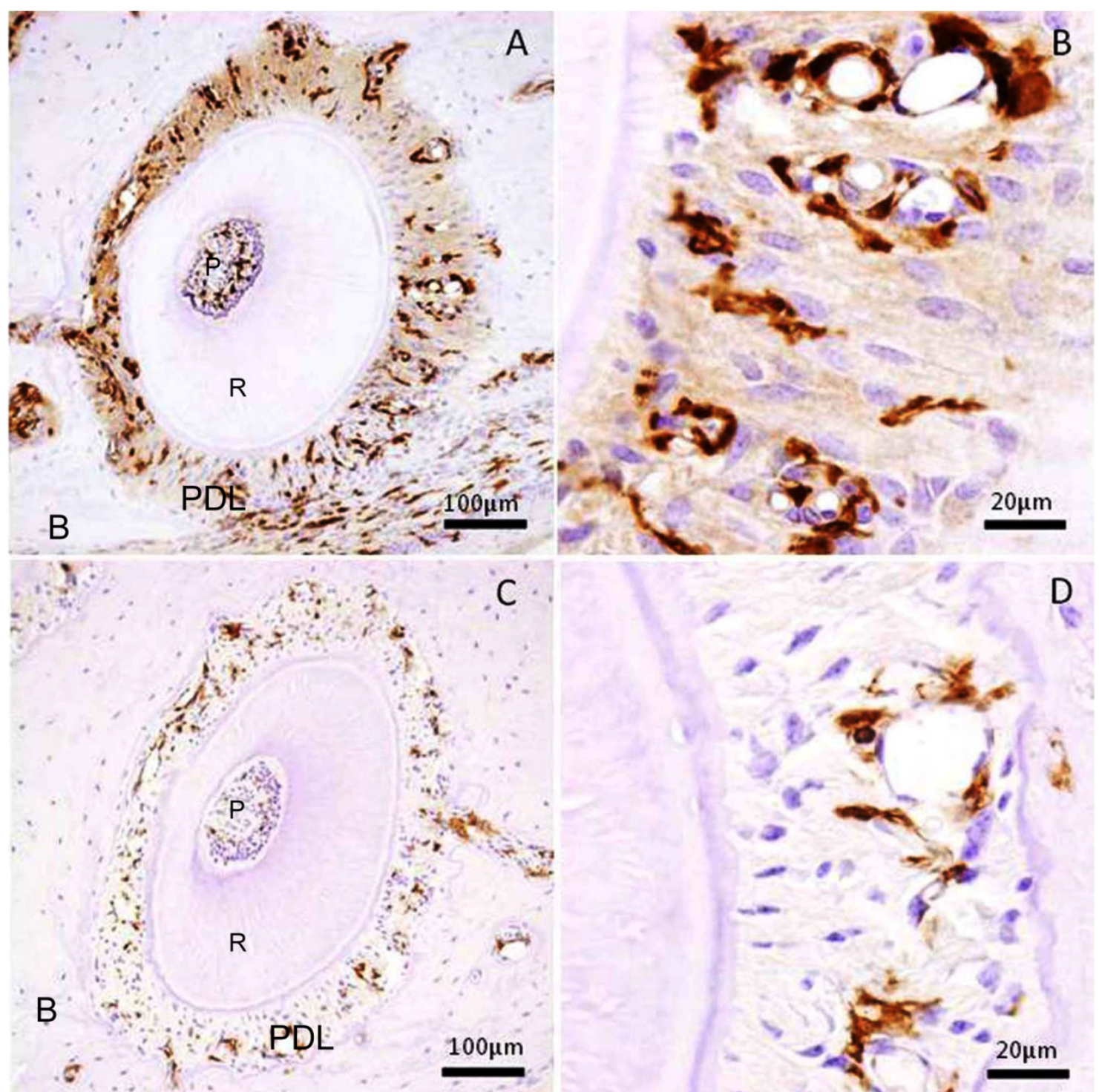

Figure I. Immunohistochemical stain for GFP in the periodontal tissue of the distal buccal root of the maxillary first molar. (A) Experimental group received mechanical stress for 5 weeks. Right side shows tension side. Left side shows pressure side. (B) High magnification of the tension side in A. (C) Control group without mechanical stress. (D) High magnification of the right side in C. P: pulp, R: root, PDL: periodontal ligament, B: maxillary alveolar bone. 


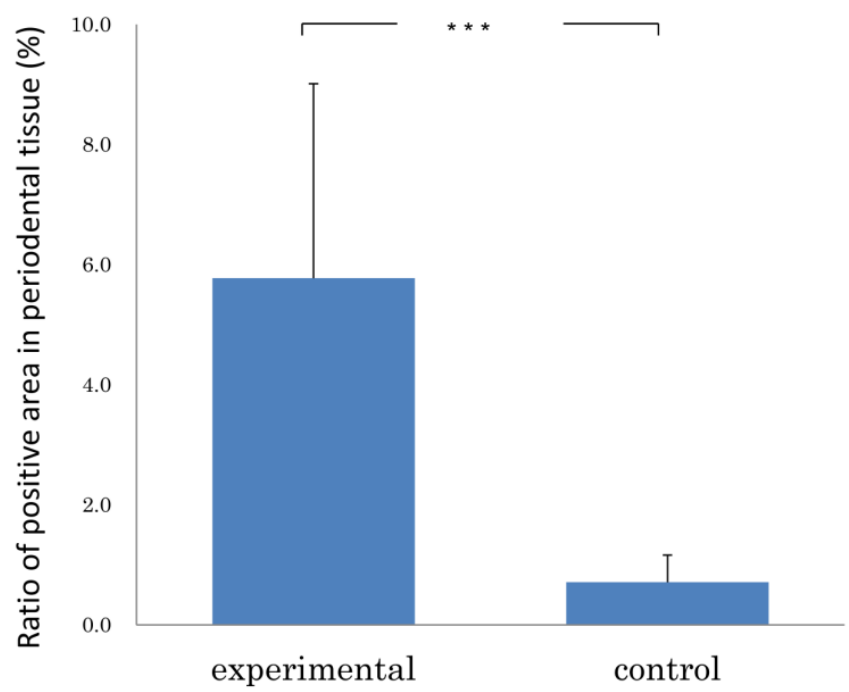

Figure 2. Ratio of positive area in periodontal tissue of experimental $(n=5)$ and control group $(n=5)$. Error bars represent the mean \pm SD. $* * * p<$ 0.00I. (Mann-Whitney U test).

In other side, using mice transplanted bone marrow cells from GFP transgenic mice, we observed GFP-positive cells within the dental pulp of mouse incisor, in the PLD, Langerhans cells in the oral epithelium, stromal fibroblasts, blood vessels and osteoclasts in the tooth region. The GFP-positive cells in the dental pulp possess arborescens processes, resembling dendritic cells and some of the odontoblast-like cells also showed positive reaction for GFP. It is clear that $\mathrm{BMC}$ possess the capacity to differentia into tooth, bone as well as connective tissue. The GFP-positive cells histopathologically differentiated into some cell types. The fluorescence immunohistochemical (IHC) and tartrate-resistant acid phosphatase (TRAP) staining techniques demonstrated these cells were detected as osteoclasts and macrophages. Furthermore, GFP-positive cells gathered adjacent blood vessels. The data suggest that GFP-positive BMC migrate into periodontal tissues and differentiate periodontal tissue component-cells.

In this study, GFP-positive cells were detected in the PDL, both in the experimental and control specimens. In the experimental group, there were numerous GFP-positive cells appearing in the experimental periodontal tissues which received intermittent stimulation of orthodontic mechanical stress. However, there were few GFP-positive cells in the control specimens. The examination group was significantly greater than that of control group, which suggests that orthodontic mechanical stress induces transplanted $\mathrm{BMC}$ in the PDL.

BMCs migrates into the jaw and differentiates into various kinds of tooth forming organ, including odontoblasts [33], osteoclasts [34] and periodontal ligament $[35,36]$. Osteoblasts and osteoclasts maintain and remodel the cancellous bone surrounding the marrow tissue. BMC has been closely involved in the repair of tissue to maintain homeostasis of the periodontal tissue by the activation of PDL fibroblasts. Moreover, external dynamic as mechanical stress strongly induces the activation of these cells. Basic principles have been established that teeth could be produced from stem cells of non-dental origin, and bone marrow stem cells are involved in the odontogenesis. As therapeutic potential in the future, these cells implant into sites of missing teeth or transplant into the bone marrow of the patient with developmental anomalies, which might lead to new approaches to tooth and jaw bone regeneration.

\section{Acknowledgments}

This study was supported in part by Grants-in Aid for Scientific Research (C) (\#23592951, \#23593075, \#25463204) and a Grant-in Aid for Young Scientists (B) (\#23792456) from the Japan Society for the Promotion of Science.

\section{Competing Interests}

The authors have declared that no competing interest exists.

\section{References}

1. Popov BV, Serikov VB, Petrov NS, et al. Lung epithelial cells induce endodermal differentiation in mouse mesenchymal bone marrow stem cells by paracrine mechanism. Tissue Eng. 2007; 13: 2441-50.

2. Zou H, Otani A, Oishi A, et al. Bone marrow-derived cells are differentially involved in pathological and physiological retinal angiogenesis in mice. Biochem Biophys Res Commun 2010; 391: 1268-73.

3. Tsujigiwa $H$, Nishizaki $K$, Teshima $T$, et al. The engraftment of transplanted bone marrow-derived cells into the olfactory epithelium. Brain Res. 2005; 1052: 10-5.

4. Bobis S, Jarocha D, Majka M. Mesenchymal stem cells: characteristics and clinical applications. Folia Histochem Cytobiol 2006; 44: 215-30.

5. Zhang HK, Zhang N, Wu LH, et al. Therapeutic neovascularization with autologous bone marrow CD34+ cells transplantation in hindlimb ischemia. Zhonghua Wai Ke Za Zhi. 2005; 43: 1275-8.

6. Tsujigiwa H, Katase N, Sathi GA, et al. Transplanted bone marrow derived cells differentiated to tooth bone and connective tissues in mice. J Hard Tissue Biol. 2011; 20: 147-52.

7. Ohazama A, Modino SA, Miletich I, et al. Stem-cell-based tissue engineering of murine teeth. J Dent Res. 2004; 83: 518-22.

8. Duailibi MT, Duailibi SE, Young CS, et al. Bioengineered teeth from cultured rat tooth bud cells. J Dent Res 2004; 83: 523-8.

9. Matsuda H, Nakano K, Muraoka R, et al. BMPs and related factors appearing in the mouse periodontal tissues due to orthodontic mechanical stress. J Hard Tissue Biol. 2010; 19: 153-60.

10. Watanabe T, Nakano K, Muraoka R, et al. Role of Msx2 as a promoting factor for Runx 2 at the periodontal tension sides elicited by mechanical stress. Eur J Med Res. 2008; 13: 425-31.

11. Watanabe T, Nakano $K$, Shimizu T, et al. Immunohistochemistry of the Pesiodontal Ligament Fibroblasts in Orthodontic Tension Sides. J Hard Tissue Biol. 2009; 18: 175-80.

12. Cho Y. A histologic study of the alveolar bone remodeling on th periosteal side incident to experimental tooth movement. JPN J Prothodontics. 1995; 54: 369-84.

13. Watanabe T, Okafuji N, Nakano K, et al. Periodontal tissue reaction to mechanical stress inmice. J Hard Tissue Biol. 2007; 16: 71-4.

14. Okabe M, Ikawa M, Kominami K, et al. Green mice as a source of ubiquitous green cells. FEBS Lett. 1997; 407: 313-9.

15. Wall DA, Hamberg SD, Reynolds DS, et al. Immunodeficiency in graft-versus-host disease. I. Mechanism of immune suppression. J Immunol. 1988; 140: 2970-6. 
16. Zijlmans JM, Visser JW, Laterveer L, et al. The early phase of engraftment after murine blood cell transplantation is mediated by hematopoietic stem cells. Proc Natl Acad Sci USA. 1998; 95: 725-9.

17. Waldo CM. Method for the study of tissue response to tooth movement. J Dent Res. 1953; 32: 690-1.

18. Watanabe T, Nakano K, Muraoka R, et al. Role of Msx2 as a promoting factor for Runx2 at the periodontal tension sides elicited by mechanical stress. Eur J Med Res. 2008; 13: 425-31.

19. Muraoka R, Nakano K, Kurihara S, et al. Immunohistochemical expression of heat shock proteins in the mouse periodontal tissues due to orthodontic mechanical stress. Eur J Med Res. 2010; 15: 475-82.

20. Huttmann A, Li CL, Duhrsen U. Bone marrow-derived stem cells and "plasticity". Ann Hematol. 2003; 82: 599-604.

21. Mezey E, Key S, Vogelsang G, et al. Transplanted bone marrow generates new neurons in human brains. Proc Natl Acad Sci USA. 2003; 100: 1364-9.

22. Sivan-Loukianova E, Awad OA, Stepanovic V, et al. CD34+ blood cells accelerate vascularization and healing of diabetic mouse skin wounds. J Vasc Res. 2003; 40: 368-77.

23. Schatteman GC, Ma N. Old bone marrow cells inhibit skin wound vascularization. Stem Cells. 2006; 24: 717-21.

24. Kalka C, Masuda H, Takahashi T, et al. Vascular endothelial growth factor (165) gene transfer augments circulating endothelial progenitor cells in human subjects. Circ Res. 2000; 86: 1198-202.

25. Ziegelhoeffer T, Fernandez B, Kostin S, et al. Bone marrow-derived cells do not incorporate into the adult growing vasculature. Circ Res. 2004; 94 : 230-8.

26. Shintani S, Murohara T, Ikeda H, et al. Augmentation of postnatal neovascularization with autologous bone marrow transplantation. Circulation. 2001; 103: 897-903.

27. Iwase $\mathrm{T}$, Nagaya $\mathrm{N}$, Fujii $\mathrm{T}$, et al. Comparison of angiogenic potency between mesenchymal stem cells and mononuclear cells in a rat model of hindlimb ischemia. Cardiovasc Res. 2005; 66: 543-51.

28. Bianco P, Riminicci M. The bone marrow stroma in vivo: Ontogeny, structure, cellular composition and changes in disease. In: Beresford JN, Owen ME, eds. Marrow Stromal Cell Culture; Handbooks in Practical animal cell biology. Combridge, UK: Cambridge University Press. 1998: 1025.

29. Caplan AI. The mesengenic process. Clin Plast Surg. 1994; 21: 429-35.

30. Jiang Y, Jahagirdar BN, Reinhardt RL, et al. Pluripotency of mesenchymal stem cells derived from adult marrow. Nature. 2002; 418: 41-9.

31. Mezey E, Chandross KJ, Harta G, et al. Turning blood into brain: cells bearing neuronal antigens generated in vivo from bone marrow. Science. 2000; 290: 1779-82.

32. Song AM, Shu R, Xie YF, et al. A study of enamel matrix proteins on differentiation of porcine bone marrow stromal cells into cementoblasts. Cell Prolif. 2007; 40: 381-96.

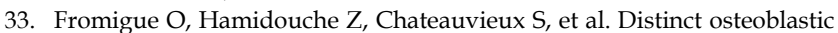
differentiation potential of murine fetal liver and bone marrow stroma-derived mesenchymal stem cells. J Cell Biochem. 2008; 104: 620-8.

34. Mancino AT, Klimberg VS, Yamamoto M, et al. Breast cancer increases osteoclastogenesis by secreting M-CSF and upregulating RANKL in stromal cells. J Surg Res. 2001; 100: 18-24.

35. Kinnaird T, Stabile E, Burnett MS, et al. Bone marrow-derived cells for enhancing collateral development. Circ Res. 2004; 95: 354-63.

36. Feng W, Madajka M, Kerr BA, et al. A novel role for platelet secretion in angiogenesis: mediating bone marrow-derived cell mobilization and homing. Blood. 2011; 117: 3893-902. 